\title{
There are similarities between rheumatic disease with lung involvement and COVID-19 pneumonia
}

\author{
Joseph Williamson $^{1} \cdot$ Louisa Black $^{2} \cdot$ Anna Black $^{3} \cdot$ Gouri Koduri $^{4} \cdot$ Clive Kelly $^{5}$ (I)
}

Received: 18 December 2020 / Accepted: 2 February 2021 / Published online: 10 February 2021

(c) Royal Academy of Medicine in Ireland 2021

\begin{abstract}
Introduction There is considerable overlap between the clinical manifestations of covid-19 pneumonia and the acute interstitial lung disease seen in certain rheumatic disorders. In addition, pulmonary fibrosis is increasingly recognised as a potentially serious consequence of both.

Methods This review explores this overlap of clinical features, risk factors and causation, offering insights into the immune mechanisms that contribute to both sets of disorders.

Results The therapeutic role of immunosuppression and biologic agents in the treatment of covid-19 is explained in the light of this.

Discussion We propose how lessons learned from the insights recently gained into each disorder can improve our insight into immunological mechanisms and application of therapeutic interventions in the other.
\end{abstract}

Keywords Biology therapy · COVID-19 pneumonia $\cdot$ Cytokine storm · Dermatomyositis · Immunosuppressives · Interstitial lung disease $\cdot$ Pulmonary fibrosis $\cdot$ Rheumatoid arthritis $\cdot$ Systemic lupus erythematosus

\section{Short commentary}

It has become apparent that there are parallels between the interstitial lung diseases (ILD) that complicate some rheumatic disorders and the pneumonitis that may accompany COVID-19 infection. This overlap extends to the epidemiologic, clinical and immunologic features of these conditions. This similarity extends from the acute manifestations of these conditions to long-term respiratory complications which includes pulmonary fibrosis as a potential consequence of COVID-19 [1]. An overlap in risk factors for COVID-19 infection and idiopathic pulmonary fibrosis, including older age, male gender, smoking, obesity and hypertension, has also been proposed [1, 2]. Abnormal lung function has been documented at discharge following

Clive Kelly

cliveryton@gmail.com

Sheffield University, Sheffield, UK

2 Walsall Hospital, Walsall, UK

3 St Thomas' Hospital, London, UK

4 Southend Hospitals, Prittlewell Chase, UK

5 University of Newcastle Upon Tyne, Newcastle upon Tyne, UK
COVID-19 infection [3] and does seem more prevalent in patients with severe disease and high levels of inflammatory markers [3, 4].

Membranous ACE2 receptors are used by the coronavirus to gain entry to human cells [5]. These metallopeptidase receptors allow viral RNA entrance into the cytoplasm of the target cells which include pneumocytes, vascular endothelial cells and cells in the proximal renal tubules. Once the virus has reached bronchioles, the main targets become bronchiolar epithelial cells and type-II ACE2+ pneumocytes. When large amounts of virions are released by infected cells, neighbouring cells become infected and viremia ensues. ACE2 expression by pulmonary cells in patients with rheumatic ILD has been the subject of recent interest [6], although there is no evidence to suggest that such patients are more prone to infection with SARS-CoV-2 itself. Other viruses are also implicated in the pathogenesis of pulmonary fibrosis and fibrosis may follow viral infection, just as it follows auto-inflammatory disease. Indeed, it appears that death following COVID-19 infection is usually due to the host's immune response, rather than to infection itself. Again, this mechanism is similar to that in many auto-immune diseases where $\mathrm{T}$ and $\mathrm{B}$ lymphocytes become hyperactive, releasing excessive cytokines that damage the patient's lungs, heart and kidneys [7]. 
The sequence of severe COVID-19 infection has been clarified [8]. The virus commonly infects older male smokers who are obese and/or diabetic [9], with the lungs the commonest organs to fail, often requiring intensive support [10]. The initial immune response to viral infection is followed by the adaptive immunity phase which decreases the viral load but may trigger release of inflammatory cytokines culminating in tissue damage and deterioration, often after apparent clinical stability. In severe infection, rises in inflammatory markers occur, with elevations in CRP, LDH, ferritin, D-dimer and IL-6 [11]. Initial symptoms do not predict prognosis, while antiviral treatment to reduce viral load must be given early to be effective. Once the adaptive immune phase begins, immunosuppression may be necessary to reduce cytokine-mediated damage. The cytokine 'storm' in COVID-19 overlaps in many manifestations with ARDS and a variety of interventions have been used to date [12].

Disproportionate numbers of deaths have occurred in men of Black or Asian minority ethnic (BAME) origin, who have a mortality 2-4 times greater than Caucasian males in the UK [13, 14] and United States (US) [15]. This is unlikely to be entirely explained by poverty, exposure or co-morbidity and may be contributed to by blood group [16]. The role of vitamin D deficiency has been investigated [17] as its deficiency increases the risk of many immunologically driven disorders [18], such as rheumatoid arthritis, where low vitamin D levels are associated with worse articular outcomes [19], the development of interstitial lung disease [20] and SLE [21]. There is increasing evidence that vitamin $\mathrm{D}$ deficiency may also be associated with an increase in both prevalence and severity of COVID-19 infection [22]. Dark skin lowers vitamin $\mathrm{D}$ exposure as does shielding indoors, so reductions in vitamin D levels may help explain why BAME people in northern latitudes are more often affected. Vitamin D levels dip in February in the northern hemisphere but peak then in the Southern [23], possibly explaining part of the reported difference in world mortality patterns.

BAME patients are also more than twice as likely to develop serious respiratory complications following infection with COVID-19, with an odds ratio against controls of 11.4 as opposed to 5.2 in Caucasian patients [24]. An ethnic prevalence trend from low to high frequency is also seen in systemic lupus erythematosus (SLE) [25, 26]. SLE occurs much more frequently and with increased severity in persons of African and Asian ancestry born and living in western countries [27]. Clinical observations support the contention that the immune system of BAME persons may be historically primed to respond more strongly when exposed to novel offending antigens and the pathophysiology of an inherent immunologically based increased inflammatory response is supported by genetic studies [28]. This may also contribute to both the higher mortality in BAME communities in the UK from COVID19 and the increased prevalence of SLE among the same populations. However, no such increased risk among the BAME population has yet been reported for rheumatic lung disease.

Clinical, radiological and pathological findings of ILD in dermatomyositis, especially in the rare subset with the antiMDA5 antibody, are very similar to those in COVID-19 [29, 30]. Clinical manifestations of acute SLE also closely mimic the pulmonary and cardiac manifestations seen following COVID-19 infection. As in some SLE patients with severe pulmonary disease [31], diffuse alveolar haemorrhage is frequently seen in the lungs of those who succumb to COVID-19 [32]. The condition is also very pro-thrombotic and post-mortem studies have consistently shown a high percentage of thrombo-occlusive lesions in the vessels and lungs, contributing to death in up to half of all cases $[33,34]$. A large US study reported $>50 \%$ reductions in mortality among ventilated patients when fully anticoagulated [35]. These observations are again analogous to those often seen in pulmonary SLE with co-existing pulmonary emboli, especially in patients with the anti-cardiolipin antibody. This antibody is also associated with a greatly increased prevalence of cardiac disease in SLE, and recent reports have shown evidence of its presence in over $50 \%$ of 172 patients with COVID-19 infection [36]. This lends further weight to the argument that all hospitalised patients with COVID-19 infection should receive standard dose prophylactic anticoagulation as currently advised by NICE (NG186). Full dose treatment should be reserved for those at higher risk as this carries the potential for significant adverse outcomes as well as benefits [37].

ILD has been reported in up to $40 \%$ established RA patients at post-mortem [38], 25\% of RA patients on HRCT [39] and carries a lifetime risk of clinical detection in $7.7 \%$ RA patients [40]. Previously, the mean survival from diagnosis for RA-ILD was just 2.6 years, although that has now improved with newer therapies [41]. ILD in RA is associated with male sex, smoking and the presence of strongly positive anti-CCP antibodies [42]. ILD can be rapidly progressive in genetically susceptible populations, especially in those of Afro-Caribbean origin. Although biologic therapy and aggressive immunomodulation have begun to improve the outlook for such patients in recent years [43], immunosuppression is certainly not likely to be of benefit in the treatment of COVID-19 because of the risk of overwhelming sepsis.

The role of the anti-B cell agent Rituximab in treating ILD in both RA and SLE is now established. Evidence suggests that if a Biologic agent is needed to treat RA in the presence of ILD, anti-TNF agents should be avoided. In those patients who are either smokers or seropositive, first-line therapy with Rituximab should be considered [43], while for seronegative patients, never smokers or those failing to respond to Rituximab, Tocilizumab has been 
recommended [44]. A recent review of the pros and cons of using Rituximab in COVID-19 argues that Rituximab should be reserved for a subset of patients with specific inflammatory complications because of the potential for reducing resistance to viral infection and the lymphopenia often consequent on such treatment [45]. However, this review suggests that where adaptive immunity might contribute to poor outcomes in COVID-19, Rituximab may well have a role.

The roles of IL- 1 and IL-6 have received much attention as the treatment of cytokine storm is one of the major issues in managing severe COVID-19 infection. High levels of IL-6 have repeatedly been reported in COVID-19 and one study of 69 patients showed that baseline IL-6 was increased in severe cases and positively correlated with higher body temperature, increased inflammatory markers and more severe findings on CT [46]. This suggests that IL-6 could also be used as a marker to monitor severity of the disease. Interleukin blockade has been shown to reduce mortality in sepsis and these findings have fuelled interest in the use of such therapy in treating the immune consequences of COVID-19 infection. IL-6 antagonism (e.g. Tocilizumab) has shown significant benefit [47]. Dexamethasone has been shown to reduce mortality in ventilated patients by a third in the RECOVERY trial [48]. It is evident that $\mathrm{T}$ cell driven cytokine release plays an essential role in the generation of progressive lung damage in both COVID-19 pneumonia and the ILD seen in certain rheumatic disorders. The 'macrophage activation syndrome' seen in patients with systemic JIA can be blocked by administration of IL-1 blocking drugs such as Anakinra [49] which may be beneficial in younger patients with COVID-19. The use of Colchicine is now being explored in the RECOVERY trial, although it has no established role in treating rheumatic lung disease.

It remains less certain as to whether the immune response to COVID-19 also involves B cells in addition to T cells. The limited evidence so far is conflicting with some patients on Rituximab recovering quickly [50], while others with more serious comorbid conditions died [51]. Further studies to assess the degree of $\mathrm{B}$ cell activation in patients with severe COVID-19 are needed. In the interim, the presence of anti-cardiolipin antibodies support the potential for B cell activation as contributing to the high prevalence of rapid onset lung and cardiac damage among susceptible individuals. If anti-nuclear antibodies and/or cyclic citrullinated peptides are also found following COVID-19 infection, anti-B cell therapy would certainly be worthy of further consideration.

There are, however, several important differences between COVID-19 and the ILD of rheumatic diseases. COVID-19 has not been reported as precipitating ILD in rheumatic disease, and several rheumatic disorders expressing high levels of IL-6, such as giant cell arteritis, are not associated with lung disease. Although there are overlaps between clinical disease expression in SLE and COVID19 with regards to cardiac manifestations and complement activation, neither tocilizumab nor sarilumab have yet been shown to alter outcomes of these complications in patients with SLE.

In conclusion, our enhanced understanding of the systemic immune response to COVID-19 infection has stimulated insights into mechanisms and therapeutic potential for the treatment of a range of interstitial lung disorders associated with auto-immune and rheumatic diseases. The important corollary has been to develop and apply our existing therapeutic knowledge to improve the prognosis of patients infected with COVID-19. The REMAP-CAP trial investigators of tocilizumab and sarilumab in COVID-19 have recently reported reductions in mortality of up to $25 \%$ : in-hospital mortality was $28 \%$ for tocilizumab and $22 \%$ for sarilumab, as compared with $35.8 \%$ for controls [52]. This confirms that IL-6 receptor antagonists do reduce mortality from COVID-19 infection in clinical practice.

\section{References}

1. Fraser E (2020) Long term respiratory complications of covid-19. BMJ 370:179

2. George P, Wells A, Jenkins G (2020) Pulmonary fibrosis and Covid-19: the potential role for anti-fibrotic therapy. Lancet Respir Med 8(8):P807-815

3. Raghu G, Wilson K (2020) Covid-19 interstitial pneumonia: monitoring the clinical course in survivors. Lancet Respir Med 8(9):P839-842

4. Das K, Lee E, Al Jawder S et al (2015) Acute Middle East Respiratory Syndrome Coronavirus: temporal lung changes observed on the chest radiographs of 55 patients. Am J Roentgenology 205(3):267-274

5. Zhang H, Penninger JM, Li Y, Zhong N, Slutsky AS (2020) Angiotensin-converting enzyme 2 (ACE2) as a SARS-CoV-2 receptor: molecular mechanisms and potential therapeutic target. Intensive Care Med 3:1-5

6. Huang H, Zhang M, Chen C et al (2020) Clinical characteristics of COVID-19 in patients with pre-existing ILD: A retrospective study in a single center in Wuhan, China. J Med Virol jmv.26174

7. Wu C, Chen X, Cai Y et al (2020) Risk factors associated with acute respiratory distress syndrome and death in patients with coronavirus disease 2019 pneumonia in Wuhan. China, JAMA Intern Med

8. Young BE, Ong SW, Kalimuddin S et al (2020) Epidemiologic features and clinical course of patients infected with SARS-CoV-2 in Singapore. JAMA 3

9. Yang X, Yu Y, Xu J et al (2020) Clinical course and outcomes of critically ill patients with SARS-CoV-2 pneumonia in Wuhan, China: a single-centred, retrospective, observational study. Lancet Respir Med. https://doi.org/10.1016/S2213-600(20)30079-5

10. Zhou F, Yu T, Du R et al (2020) Clinical course and risk factors for mortality of adult inpatients with COVID-19 in Wuhan, China: a retrospective cohort study. Lancet. https://doi.org/10.1016/ S0140-6736(20)30566-3 
11. Mehta P, McAuley DF, Brown M et al (2020) COVID-19: consider cytokine storm syndromes and immunosuppression. Lancet 395(10229): 1033-1034

12. Yessayan L, Szamosfalvi B, Napolitano L et al (2020) Treatment of Cytokine Storm in COVID-19 patients with immunomodulatory therapy. ASAIO J 66(10):1079-1083. https://doi.org/10.1097/ MAT.0000000000001239

13. Lassale C, Gaye B, Hamer M et al (2020) Ethnic disparities in hospitalisation for covid-19 in england: the role of socioeconomic factors, mental health, and inflammatory and pro-inflammatory factors in a community-based cohort study

14. Booth R, Barr C (2020) Black people four times more likely to die from Covid-19 ONS finds. https://www.theguardian.com/world/ 2020/may/07/black-people-four-times-more-likely-to-die-fromcovid-19-ons-finds

15. Bentley GR (2020) Don't blame the BAME: Ethnic and structural inequalities in susceptibilities to COVID-19. Am J Hum Biol. 32:e23478. https://doi.org/10.1002/ajhb.23478

16. Ellinghaus D, Degenhardt F, Bujanda L et al (2020) The ABO blood group locus and a chromosome 3 gene cluster associate with SARS-CoV-2 respiratory failure in an Italian-Spanish genome-wide association analysis. medRxiv. https://doi. org/10.1101/2020.05.31.20114991

17. Alipio M (2020) Vitamin D supplementation could possibly improve clinical outcomes of patients infected with Coronavirus-2019 (COVID19). Available at SSRN: https://ssrn.com/abstract=3571484 or https:// doi.org/10.2139/ssrn.3571484

18. Agmon-Levin N, Theodor E, Segal R, Shoenfeld Y (2013) Vitamin D in systemic and organ-specific autoimmune diseases. Clin Rev Allergy Immunol 45(2):256-266. https://doi.org/10.1007/s12016-012-8342-y

19. Kiran G, Debashish D (2008) Vitamin D and rheumatoid arthritis: is there a link? Int J Rheum Dis 20. https://doi.org/10.1111/j.1756185X.2008.00385.x

20. Sherin H, Dalia E, Diab H, Takwa Y (2019) Vitamin D deficiency and pulmonary affection in rheumatoid arthritis. Egyptian J Chest Dis Tuberc 68(4). https://doi.org/10.4103/ejcdt.ejcdt_65_19

21. Guan SY, Cai HY, Wang P et al (2019) Association between circulating 25-hydroxyvitamin $\mathrm{D}$ and systemic lupus erythematosus: A systematic review and meta-analysis. Int J Rheum Dis. https://doi. org/10.1111/1756-185X.13676

22. Benskin L (2020) A basic review of the preliminary evidence that COVID-19 risk and severity is increased in vitamin d deficiency. Front Public Health 8. https://doi.org/10.3389/fpubh.2020.00513

23. Bolland MJ, Grey AB, Ames RW et al (2007) The effects of seasonal variation of 25-hydroxyvitamin $\mathrm{D}$ and fat mass on a diagnosis of vitamin D sufficiency. Am J ClinNutr 86:959-964

24. Ayoubkhani D, Khunti K, Nafilyan V et al (2021) Epidemiology of post-COVID syndrome following hospitalisation with coronavirus: a retrospective cohort study. medRxiv preprint. https://doi. org/10.1101/2021.01.15.21249885

25. Bae S-C, Fraser P, Liang MH (1998) The epidemiology of systemic lupus erythematosus in populations of African ancestry: a critical review of the "prevalence gradient hypothesis." Arthritis Rheum 41(12):2091-2099

26. Molokhia M, McKeigue P (2000) Risk for rheumatic disease in relation to ethnicity and admixture. Arthritis Res 2(2):115-125

27. Sibanda EN, Chase-Topping M, Pfavayi LT et al (2018) Evidence of a distinct group of Black African patients with systemic lupus erythematosus. BMJ Glob Heal 3(5):e000697

28. Nédélec Y, Sanz J, Baharian G et al (2016) Genetic ancestry and natural selection drive population differences in immune responses to pathogens. Cell 167(3):657-669.e21

29. De Lorenzis E, Natalello G, Gigante L et al (2020) What can we learn from rapidly progressive interstitial lung disease related to anti-MDA5 dermatomyositis in the management of COVID-19? Autoimmun Rev 19(11):102666
30. Megremis S, Walker T, He X et al (2020) Antibodies against immunogenic epitopes with high sequence identity to SARS-CoV-2 in patients with autoimmune dermatomyositis. Ann Rheum Dis. https://doi.org/10.1136/annrheumdis-2020-217522

31. Santos-Ocampo AS, Mandell BF, Fessler BJ (2000) Alveolar hemorrhage in systemic lupus erythematosus. Chest 118(4):1083-1090

32. Guan WJ, Ni ZY, Hu Y et al (2020) Clinical characteristics of coronavirus disease 2019 in China. N Engl J Med 382:1708-1720. https://doi.org/10.1056/NEJMoa2002032

33. Argulian E, Sud K, Vogel B et al (2020) Right ventricular dilation in hospitalized patients with COVID-19 infection. JACC Cardiovasc Imag. https://doi.org/10.1016/j.jcmg.2020.05.010

34. Lax S, Skok K, Zechner P et al (2020) Pulmonary arterial thrombosis in COVID-19 with fatal outcome: Results from a prospective, singlecentre clinicopathologic case series. Ann Intern Med

35. Paranjpe I, Fuster V, Lala A et al (2020) Association of treatment dose anticoagulation with in-hospital survival among hospitalized patients with COVID-19. J Am CollCardiol. https://doi. org/10.1016/j.jacc.2020.05.001

36. Zuo Y, Estes SK, Ali RA et al (2020) Prothrombotic autoantibodies in serum from patients hospitalized with COVID-19. Sci Transl Med 570. https://doi.org/10.1126/scitranslmed.abd3876

37. McBane R, Roldan V, Niven A et al (2020) Anticoagulation in COVID-19: a systematic review, meta-analysis, and rapid guidance from Mayo Clinic. Mayo ClinProc 95(11):2467-2486

38. Ellman P, Ball RE (1948) Rheumatoid disease with joint and pulmonary manifestations. BMJ 2(4583):816-820

39. McDonagh J, Greaves M, Wright AR et al (1994) High resolution computed tomography of the lungs in patients with rheumatoid arthritis and interstitial lung disease. Br J Rheumatol 33: 118-122

40. Bongartz T, Nannini C, Medina-Velasquez Y et al (2010) Incidence and mortality of interstitial lung disease in rheumatoid arthritis: a population-based study. Arthritis Rheum 62(6):1583-1591

41. Kelly CA, Nisar M, Arthanari S et al (2020) Rheumatoid arthritis related interstitial lung disease - improving outcomes over 25 years: a large multicentre UK study. Rheumatol (Oxford, England). https:// doi.org/10.1093/rheumatology/keaa577

42. Kelly CA, Saravanan V, Nisar M et al (2014) Rheumatoid arthritisrelated interstitial lung disease: associations, prognostic factors and physiological and radiological characteristics-a large multicentre UK study. Rheumatol 53: 1676-1682

43. Kelly CA (2014) Interstitial lung disease in rheumatoid arthritis. Rheumatol 53(suppl_1):i15. https://doi.org/10.1093/rheumatology/ keu066.002

44. Manfredi A, Cassone G, Furini F et al (2020) Tocilizumab therapy in rheumatoid arthritis with interstitial lung disease: a multicentre retrospective study. Intern Med J 50(9):1085-1090

45. Mehta P, Porter J, Chambers R et al (2020) B-cell depletion with rituximab in the COVID-19 pandemic: where do we stand? Lancet Rheumatol 2(10):e589-e590

46. Liu T, Zhang J, Yang Y et al (2020) The potential role of IL-6 in monitoring severe case of coronavirus disease 2019. medRxiv. https:// doi.org/10.1101/2020.03.01.20029769

47. Xu X, Han M, Li T et al (2020) Effective treatment for severe COVID-19 patients with tocilizumab. ChinaXiv:20200300026

48. The RECOVERY Collaborative Group (2020) Dexamethasone in hospitalized patients with Covid-19 - preliminary report. N Engl J Med. https://doi.org/10.1056/NEJMoa2021436

49. Balkhair A, Al-Zakwani I, Al BM et al (2020) Anakinra in hospitalized patients with severe COVID-19 pneumonia requiring oxygen therapy: results of a prospective, open-label, interventional study. Int J Infect Dis 17(103):288-296. https://doi.org/10.1016/j. ijid.2020.11.149 
50. Fallet B, Kyburz D, Walker U (2020) Mild course of COVID-19 and spontaneous virus clearance in a patient with depleted peripheral blood B cells due to rituximab treatment. Arthritis Rheumatol 72(9):1581-1582

51. Tepasse PR, Hafezi W, Lutz M et al (2020) Persisting SARS-CoV-2 viraemia after rituximab therapy: two cases with fatal outcome and a review of the literature. Brit J Haematol 190(2):185-188

52. Gordon A, Mouncey P, Al-Beidh F et al (2020) Interleukin-6 receptor antagonists in critically ill patients with Covid-19 preliminary report: the REMAP-CAP investigators. medRxiv preprint. https://doi.org/10.1101/2021.01.07.21249390 